\title{
FOCES - a fibre optics Cassegrain échelle spectrograph ${ }^{\star}$
}

\author{
M.J. Pfeiffer, C. Frank, D. Baumüller, K. Fuhrmann, and T. Gehren \\ Institut für Astronomie und Astrophysik der Universität München, Scheinerstr. 1, 81679 München, Germany \\ e-mail: pfeiffer@usm.uni-muenchen.de, fuhrmann@usm.uni-muenchen.de, gehren@usm.uni-muenchen.de
}

Received December 12; accepted June 26, 1997

\begin{abstract}
We have designed and built the échelle spectrograph FOCES fed by $100 \mu \mathrm{m}$ optical fibres to be mounted at the Cassegrain focus of either the $2.2 \mathrm{~m}$ or the $3.5 \mathrm{~m}$ telescope at the Calar Alto Observatory. The spectrograph itself follows a white-pupil design collimated with two off-axis parabolic mirrors. The $15 \mathrm{~cm}$ beam leaving the 31.6 lines/mm R2 échelle is refocussed in the vicinity of a small folding mirror which allows efficient removal of scattered light. The cross-dispersion is achieved with a tandem prism mounting, and the beam imaged with an $f / 3$ transmission camera onto a field centered on a $1024^{2}$ thinned Tektronix CCD with $24 \mu \mathrm{m}$ pixel diameter. The échelle image covers the visible spectral region from 380 to $750 \mathrm{~nm}$ displayed in 70 spectral orders with full spectral coverage. Spectral orders are separated by 20 pixels in the blue and by 10 pixels in the red. The maximum spectral resolution is $R=\lambda / \Delta \lambda=40600$ with a 2 pixel resolution element; unvignetted resolution as defined by the fibre alone would be obtained at $R=18000$. Replacing the CCD by a $2048^{2}$ chip with $15 \mu$ m pixel diameter and taking into account light losses from a reduced entrance slit width a full 2 pixel resolution of $R=65000$ is obtained.

The above concept has made FOCES an extremely well-defined instrument. A number of successful test installations at the Cassegrain foci of the Wendelstein $80 \mathrm{~cm}$ telescope, the Calar Alto $2.2 \mathrm{~m}$ and $3.5 \mathrm{~m}$ telescopes has produced spectra of high quality for up to $60 \mathrm{~min}$ exposures. The limiting magnitude for a $1 \mathrm{hr}$ exposure with an $S / N$ ratio of 100 scales to $V=12$ for a $3.5 \mathrm{~m}$ telescope which is only slightly less than expected from laboratory tests. In an alternative mode FOCES offers a second fibre carrying the sky background signal to correct extremely faint object spectra. This mode obtains the required higher cross-dispersion from an additional grism resulting in a correspondingly reduced spectral coverage.
\end{abstract}

Send offprint requests to: M. Pfeiffer (also download from http://usm.uni-muenchen.de/papers.html

* Based in part on observations collected at the GermanSpanish Astronomical Center, Calar Alto, Spain.
Key words: instrumentation: spectrographs - methods: observational — methods: data analysis — techniques: spectroscopic

\section{Introduction}

Many large telescopes provide a Coudé focus to observe high-resolution stellar absorption line spectra in a format that was originally fitted to the size of photographic plates. While the photographic emulsion since then has been replaced by the CCD, the available spectrographs often do not allow the exposure of more than a very small part of a single spectral order. This requires multiple exposures as soon as a significant fraction of the visible spectrum is needed, either introducing a strong decrease of available telescope time or leading to an unacceptable shortening of the scientific programs.

The obvious solution is an échelle spectrograph installed at either Coudé or Cassegrain focus. Due to the surprising development of modern CCDs cross-dispersed échelle spectrographs can image the complete visible spectrum with moderate to high resolution on a single chip of $1024^{2}$ or even $2048^{2}$ pixels. A few échelle spectrographs have been built during the last decade and are now used with great success for a number of astrophysical programs. There are, however, large telescopes not equipped with an échelle spectrograph though there exists an increasing demand of spectroscopic telescope observing time. One of these sites was the German-Spanish Astronomical Center on the Calar Alto with its large telescopes of $2.2 \mathrm{~m}$ and $3.5 \mathrm{~m}$ diameter. The $2.2 \mathrm{~m}$ telescope which is a twin to the one on La Silla, has a large Coudé spectrograph with $f / 3$ and $f / 12$ cameras. Both can be equipped with a CCD camera providing resolution elements (2 pixel) of $\lambda / \Delta \lambda=20000$ to 45000 . The shorter camera is therefore not really appropriate to observe absorption line spectra of cool stars. Moreover, the CCD installed at the $f / 3$ focus cuts out a small spectral range of $\sim 10 \mathrm{~nm}$ only. 
For many purposes this is unsatisfactory. Abundance analyses or simultaneous investigations of spectral lines always require significant spectral coverage which could be obtained only with multiple exposures. An illustrative example is the Coudé spectroscopy of a 10th magnitude star. Near $5000 \AA$ a single exposure of $60 \mathrm{~min}$ is required to reach a $S / N$ ratio of 100 . If a total of $1000 \AA$ coverage is necessary one had to spend a whole night observing only this one star. Vice versa, a large amount of observing time can be saved by a spectrograph with resolution comparable to Coudé spectrographs but spectral coverage including the full visible wavelength range. We therefore decided to build an échelle spectrograph for use at the Calar Alto $2.2 \mathrm{~m}$ and $3.5 \mathrm{~m}$ telescopes for which we benefitted strongly from experience with existing échelle spectrographs such as the ESO CASPEC which was one of the few instruments being in regular use at large telescopes.

The final design of this instrument was driven by a number of constraints that are specified by the needs of the spectroscopy of stellar absorption lines,

- The definition and stability of the échelle image on currently available CCDs was a fundamental requirement. We used fibre optics to feed the spectrograph, which can thus be mounted in a thermally and mechanically isolated environment to provide an exceptionally stable echelle order image on the CCD. This allows a more accurate definition of spectrum positions than is possible for a spectrograph mounted at the telescope.

- A major problem encountered with existing échelle spectrographs such as the CASPEC is the different $i l$ lumination of the spectrograph entrance slit for a stellar image and for the flatfield lamp. Most of that difference is removed by scrambling the light in the fibre.

- Scattered light background under uncontrolled conditions often constitutes a substantial fraction of the astronomical light. Such losses also tend to degrade the information contained in spectral orders. This has been discussed in a number of papers (Gehren \& Ponz 1986; Hall et al. 1994). We found it much better to remove most of the straylight from the camera beam with the help of an intermediate slit. Replacing the usual crossdispersion grating by a prism also keeps the straylight at a minimum.

- As discussed above the flatfield correction of échelle order spectra is problematic. Small drifts of order positions as observed in some échelle spectrographs mounted at the Cassegrain focus lead to a situation in which the order centers of astronomical object and flatfield exposure are systematically displaced, and a pixel-to-pixel flatfield calibration becomes impossible. Our solution to that problem is a combination of a white light flatfield resulting from an illuminated screen in front of the échelle and the usual one-dimensional order flatfield.
- Spectral coverage and adjacent order overlap are constraints to the échelle format. Spectral coverage has been one of the driving motivations to build FOCES. High-resolution stellar spectroscopy is dedicated to cool stars. The flux of such objects is heavily weighted towards the red which accounts for long exposure times to obtain a sufficient signal in the blue. During such exposures the red spectrum becomes overexposed and therefore useless. Depending on the type of crossdisperser the échelle orders become very crowded in the red or blue region of the spectrum, and this also constrains spectral coverage. Finally, large spectral coverage requires a highly efficient atmospheric dispersion corrector. We therefore decided to limit FOCES to a standard 380 to $750 \mathrm{~nm}$ coverage, with an option to shift the spectral range towards 430 to $900 \mathrm{~nm}$. Implications for the selection of optical components, fibres and the CCD are discussed below.

- The use of optical fibres to transmit the astronomical light from the telescope focus to a remote spectrograph entrance slit has led to some experience during recent installations (Ramsey \& Huenemoerder 1987). It is a source of potential light losses. Compared with a system of lenses and mirrors the throughput is inferior. However, The light-scrambling properties of optical fibres lead to an improved illumination of the spectrograph entrance slit, and this allows more accurate Doppler velocity determinations (Heacox 1986; Ramsey 1988). The disadvantages of fibre spectroscopy due to degradation and transmission properties may lead to a significant reduction of the performance in the near infrared, although more recent developments have led to considerably improved properties (Barden 1995).

We give a full description of the spectrograph in Sect. 2 including optical layout, electronic and mechanical systems. In Sect. 3 we discuss the telescope module and the fibre optics. In Sect. 4 we present some of the first observations obtained at the $2.2 \mathrm{~m}$ telescope of the GermanSpanish Astronomical Center on Calar Alto. We present a data extraction software used to obtain échelle order spectra and compare the results of these test observations with the predicted performance of FOCES. The final section carries a discussion of the spectrograph in its present state.

\section{Spectrograph}

The principles discussed in the introduction define FOCES as a stationary échelle spectrograph mounted at some remote laboratory in the dome building. This isolation of the échelle spectrograph is of threefold nature:

- first of all the spectrograph is $\approx 18 \mathrm{~m}$ away from the Cassegrain focus of the telescope. The interruption of the optical light path therefore requires a relay in form 
of an optical fibre with advantages and disadvantages discussed above;

- the spectrograph is also isolated mechanically. This has two advantages: it decouples the instrument from the movement of the telescope and thus avoids mechanical flexure. It also provides the base for an efficient reduction of mechanical vibrations;

- finally, it puts the spectrograph in a controlled thermal environment. Consequently, thermal drifts of the instrument during an observing night due to temperature changes in the dome are avoided. The spectrograph can be isolated, first by holding the temperature in the laboratory constant, and second by controlling the heat development in the spectrograph itself if necessary. In fact, the latter control proved unnecessary since encoders and motors were selected to produce minimum heat.

This concept has only two severe disadvantages. The optical fibre link will swallow photons that in a direct mounting of the instrument would reach the detector, and our fibre design does not allow a long slit that may be needed for sky background correction of very faint objects. Both disadvantages are taken into account. The light losses due to fibre transmission (see below) are more than compensated by the possibility to use a transmission camera instead of the typical Schmidt camera of a directly mounted instrument. Moreover, FOCES is intentionally designed for a $S / N \geq 30$ which normally limits the sky background to a small fraction of the total signal $(\leq 10 \%)$.

FOCES is not really a high-resolution spectrograph. Budgetary and technical restrictions forced us to compromise the astronomical requirements with beam size, fibre diameters and available CCD chips; therefore the échelle spectrograph will work at the $2.2 \mathrm{~m}$ telescope with a standard resolution of $R=40600$ providing a two-pixel resolution element. This limit is set by the $24 \mu \mathrm{m}$ pixel distance and the area of the $1024^{2}$ Tektronix CCD chip. The maximum spectrograph resolution product at the $2.2 \mathrm{~m}$ telescope is $R \phi=60300$ with the slit width $\phi$ entered in arcsec; at the $2.2 \mathrm{~m}$ telescope the standard slit width corresponds to $130 \mu \mathrm{m}$ which subtends a 1.5 arcsec angle on the sky. Thus a CCD chip with $2048^{2}$ pixels of $15 \mu \mathrm{m}$ distance would yield a 2 pixel resolution of $R=65000$ with increased spectral coverage, however, implying significant light losses at the entrance slit due to reduction to 0.8 arcsec slit width. The spectrograph is also designed to be mounted alternatively at either the Calar Alto $2.2 \mathrm{~m}$ or the $3.5 \mathrm{~m}$ telescope, however, with a reduced throughput or resolution at the $3.5 \mathrm{~m}$.

\subsection{Optical design}

The optical layout of the FOCES spectrograph follows a white pupil design that has been documented by Baranne (1988). The advantages are discussed there in detail so we
Table 1. Basic FOCES data

$\begin{array}{lll}\text { optical bench } & & 2400 \times 900 \times 203 \mathrm{~mm} \\ \text { collimator } & \text { focal length } & 1524 \mathrm{~mm} \\ \text { diameter } & 254 \mathrm{~mm} \\ \text { échelle grating } & \text { size } & 165 \times 320 \times 50 \mathrm{~mm} \\ & \text { blaze angle } & 65^{\circ} \\ \text { cross-disperser } & \text { grating constant } & 31.6 \mathrm{lines} / \mathrm{mm} \\ & \text { size } & 190 \times 160 \times 112.6 \mathrm{~mm} \\ \text { camera } & \text { angle of deviation } & \approx 42^{\circ} \\ & \text { focal length } & 455 \mathrm{~mm}(\mathrm{f} / 3)\end{array}$

need only emphasize that one of its important features for us is the intermediate image of the spectrum which can efficiently be cleaned from scattered light emanating from the échelle grating. Baranne et al. (1996) have built a very similar instrument (ELODIE) for the Observatoire de Haute-Provence. It uses a $\tan \theta=4$ échelle grating in combination with a $10 \mathrm{~cm}$ spectrograph beam and a prismgrism cross-disperser. Due to the strong support obtained from the European Southern Observatory the optical layout of FOCES emerged to become very similar to the one proposed for the UV-Visual Echelle Spectrograph built for the ESO Very Large Telescope (Dekker et al. 1992). The optical characteristics of the FOCES design are given in Table 1, and the layout is shown in Fig. 1. A few principles differ from those specified for the UVES.

1. The area of the entrance slit is immediately aside the folding mirror which makes the UVES-type entrance folding mirror unnecessary. The entrance slit and its spectral image are therefore very near together.

2. The spectrograph beam is only $15 \mathrm{~cm}$, therefore the échelle grating is correspondingly smaller, with an R2 échelle fitting the goals of FOCES.

3. Instead of a low-order grating we use a double prism for cross-dispersion. This accounts for a less strongly changing interorder distance, and it significantly reduces local straylight in the spectrum.

4. Finally, FOCES has a transmission camera of an only moderate $f / 3$ design.

\subsubsection{Echelle grating}

The échelle grating is blazed at $\phi_{B}=65^{\circ}$ and has 31.6 lines $/ \mathrm{mm}$. Its ruled area corresponds to an overfilling by the $15 \mathrm{~cm}$ beam of $34 \mathrm{~mm}$. This $11 \%$ linear overfilling leads to only $4.5 \%$ vignetting of the beam which optimizes the product of throughput and resolution. As is evident from Fig. 1 the spectrograph works in an extreme nearLittrow configuration of the échelle which is possible with a non-zero angle transverse to the direction of dispersion, $\gamma=0.7^{\circ}$. The obvious advantages of the quasi-Littrow configuration (cf. Schroeder 1987) include an increased efficiency of the échelle grating.

The échelle is kept in a gimbal mount with all three axes fully adjustable as shown in Fig. 2a. It is oriented 


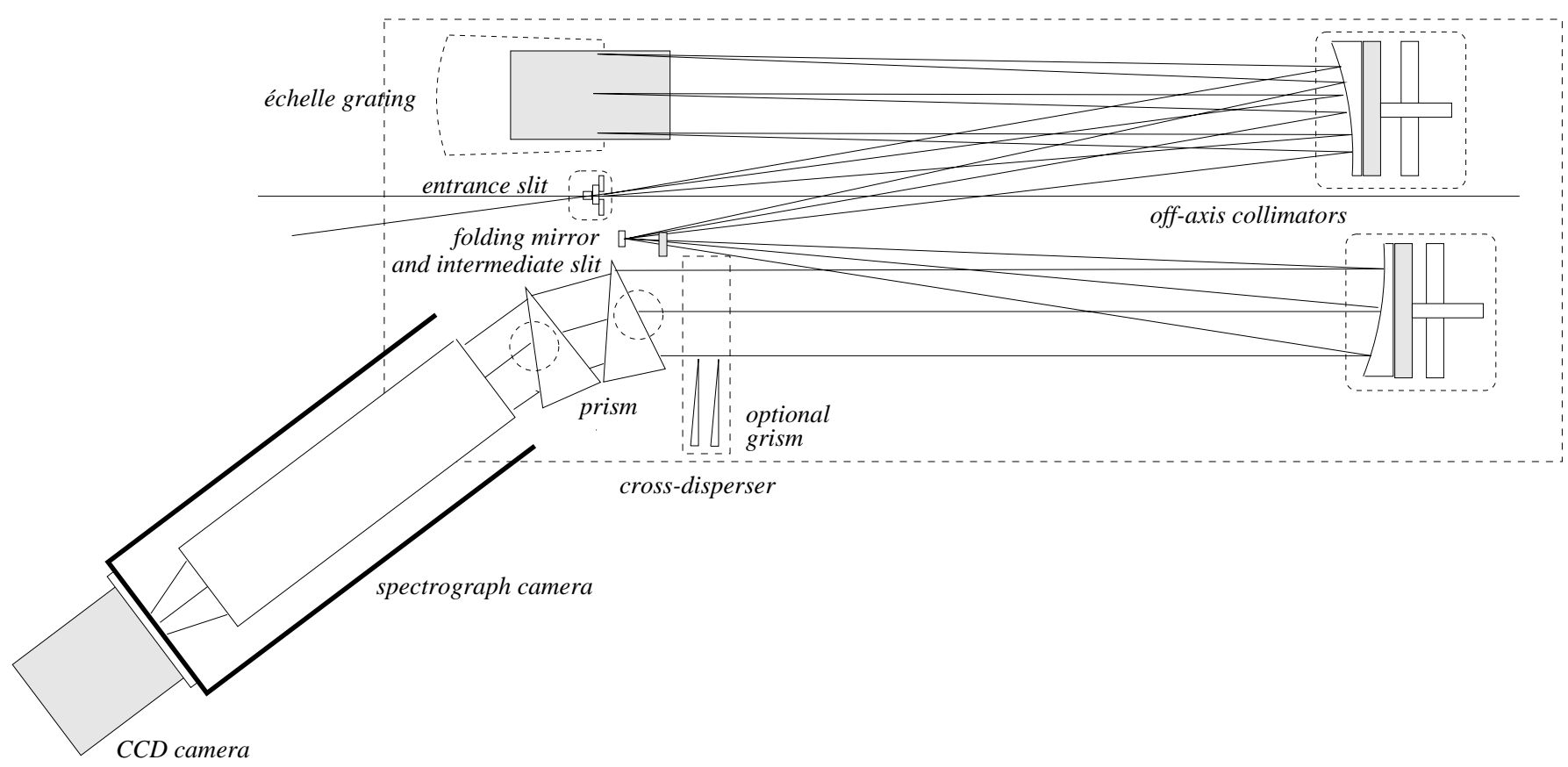

Fig. 1. Optical layout of the FOCES spectrograph with entrance slit, collimators, échelle grating, folding mirror, prism/grism cross-disperser, and camera

upside down to avoid dust contamination of the surface. The adjustment is accurate to within \pm 10 arcsec on each of the axes. Two of the axes are controlled manually with screws, while the transverse horizontal axis can be read out and moved with a DC motor using a gray code absolute encoder. This allows a careful centering of the blaze function on the detector. Intentionally, it also allows decentering the orders such as to cover near-infrared spectral regions at the order ends that are outside normal coverage (i.e. at wavelengths $>750 \mathrm{~nm}$ where the full spectral coverage ends).

\subsubsection{Collimators and folding mirror}

The collimator consists of a pair of off-axis paraboloids cut from a single parabolic $f / 2$ Zerodur $^{\mathrm{TM}}$ blank with a focal length of $1524 \mathrm{~mm}$. The diameter of the collimators is $254 \mathrm{~mm}$, and they are mounted in aluminium housings that allow directional fine tuning with precision screws. For optimal performance the mirror surfaces are silvercoated with a special cover avoiding corrosion. The overall reflectivity is more than $99 \%$ at maximum (near $6000 \AA$ ), but decreases to $90 \%$ near $4000 \AA$. The double paraboloid arrangement is very efficient in removing most of the aberrations due to the tilted incidence of the beam (see Dekker et al. 1992).

Between the two collimators the spectrograph beam is folded on a small plane Zerodur ${ }^{\mathrm{TM}}$ mirror of $100 \times 10 \mathrm{~mm}$ size, in the immediate vicinity of which an intermediate image of the échelle spectrum is observed. This offers the unique advantage to free the spectrum from most of the scattered light produced at the échelle and other surfaces and edges simply by inserting a diaphragm which passes only the light falling through the intermediate image. The resulting improvement gives FOCES nearly the quality of a monochromator as is shown in Sect. 4.

\subsubsection{Cross disperser}

After recollimation on the second collimator the beam enters a cross-dispersing tandem prism (cf. Fig. 2b). The prisms are made of LF5 with a basis length of $160 \mathrm{~mm}$ and a width of $112.6 \mathrm{~mm}$. LF5 has been chosen because its transmission in the near ultraviolet is very high as compared with other flint glasses, and because its angular dispersion is high enough. The prism angle is $33^{\circ}$, and the prisms are used near minimum deviation. The strong cross-dispersion required implies the tandem prism arrangement to avoid problems with total reflexion that would be present on a single $55^{\circ}$ prism. The symmetric position is read out and adjusted with an accuracy of 2 arcmin.

The prisms are anti-reflection coated with an efficiency better than $95 \%$ over the full visible wavelength region. Compared with a grating as a cross-disperser the tandem prism thus has a gain in efficiency of approximately 20 to $30 \%$. It also removes the global cross-order scattered light level on the detector. Moreover, the prism is also first choice because it allows a much more economic use of the CCD real estate; whereas a grating produces an order separation $\Delta y \propto \lambda^{2}$ the prism order separation varies only $\propto \lambda^{-1}$ clustering in the red instead of the blue. The second 


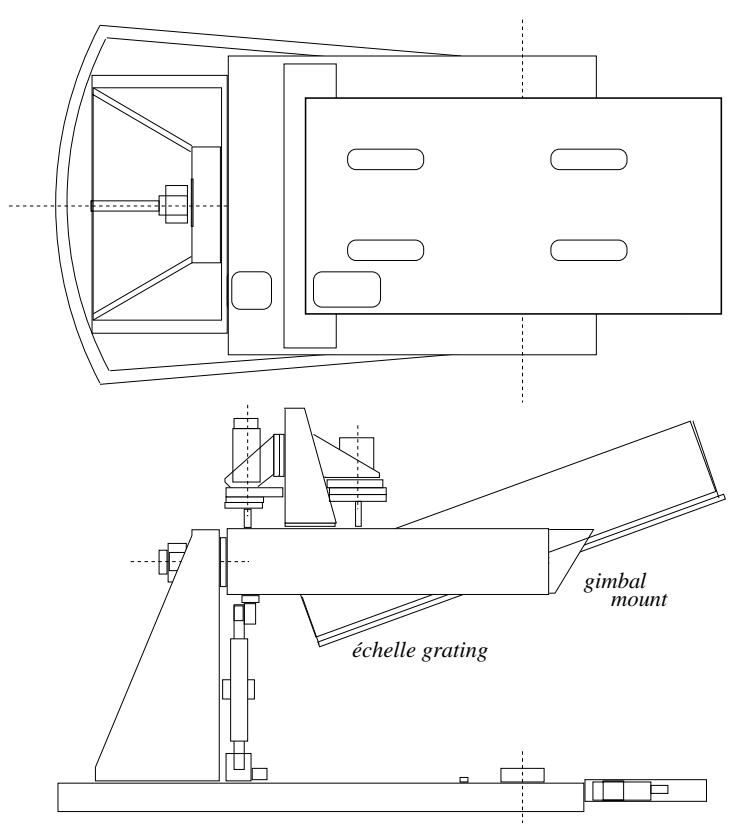

(a)

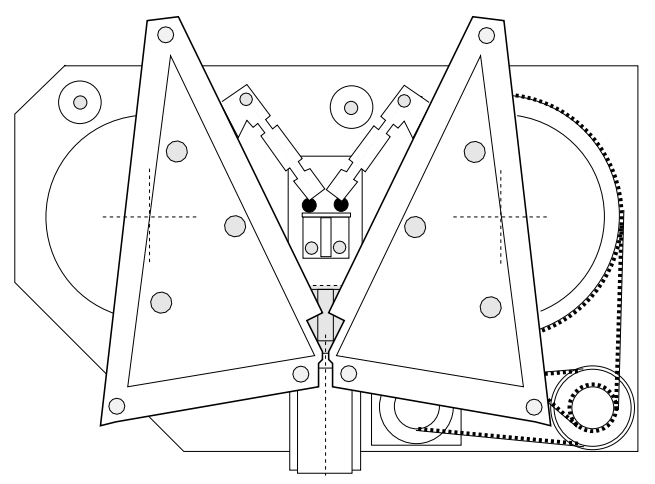

LF5 standard prism cross-disperser on tandem mounting

(b)

(c)

Fig. 2. Spectrograph components: a) échelle mounting from above (top) and from aside (bottom), b) mounting of cross-disperser tandem prism used for single-fibre mode, c) mounting of blue and red grism used in combination with the prisms for higher cross-dispersion in dual-fibre mode

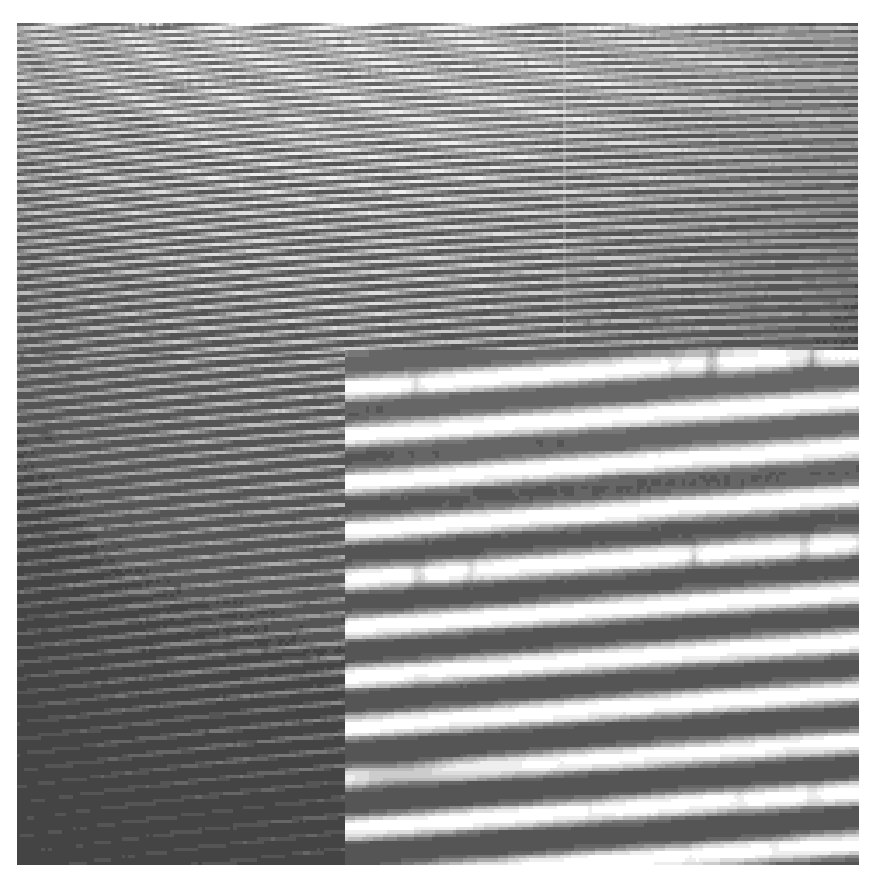

Fig. 3. Full view of recorded single-fibre spectrum of a cool star displaying 70 échelle orders ranging from 3900 to $7000 \AA$, with a single bad column on the CCD chip of the test camera. At bottom right the inset shows a magnified section of the échelle image near the atmospheric $\mathrm{O}_{2}$ band at $6800 \AA$. The spectrograph slit is set to full two-pixel resolution $(R=40600)$, corresponding to $1.5^{\prime \prime}$ at the focal plane. The seeing was $\approx 2.5^{\prime \prime}$ as is evident from the cross-order width aspect is particularly important since the blue signal is always considerably fainter than the red and the interorder definition therefore much more significant.

While the prism cross-disperser provides a sufficient separation of the échelle orders for observations with a single fibre, FOCES can also be used in a dual-fibre mode that requires nearly twice the cross-dispersion. This is achieved with an additional grism that can be moved into the beam immediately in front of the prisms as shown in Fig. 2c. The resulting échelle image pattern is shown in Fig. 3 for a short single-fibre exposure of Procyon.

\subsection{Mechanical design and stability}

The spectrograph is mounted on an optical bench made of ferromagnetic stainless steel which weighs $\approx 300 \mathrm{~kg}$. While its mechanical properties are optimized for frequencies below $50 \mathrm{~Hz}$ to compensate for small perturbations such as encountered in dome buildings, there are resonances near $140 \mathrm{~Hz}$ and higher, which are damped pneumatically using a system of shock absorbers to mount the bench on. The overall mechanical stability is therefore very high and makes the spectrograph particularly useful for radial velocity work.

Thermal stability is enforced by the design itself. FOCES is set up under controlled thermal conditions such as found in a Coudé laboratory of a telescope dome. The room temperature is not assumed to vary by more than $2 \mathrm{~K}$. The spectrograph with the optical bench is housed in a cover additionally isolated with polystyrene. No active 
cooling of the spectrograph is needed since the internal heat from motors is negligibly small; this is guaranteed by switching off the active electronic components immediately after they have been used; this is possible due to use of absolute encoders.

\section{Fibre optics and telescope module}

The coupling of telescope and remote spectrograph by optical fibres is a relatively new technique. Not all the problems and their consequences for scientific applications are by now fully understood; therefore it is necessary to test the fibre characteristics such as transmission and degradation as well as their imaging properties and their response to mechanical and thermal conditions. Provided that the latter two items are well under control it is transmission and degradation which must be optimized with respect to the optical and astronomical requirements.

\subsection{Properties of optical fibres}

Although the basic properties of optical fibres used in astronomy have been examined repeatedly (e.g. Ramsey 1988), there seem to co-exist diverging results about the quantitative performance of different fibre types. We have therefore measured degradation and transmission properties of various fibres from different distributors, among them Laser Components, Mitsubishi, Polymicro, and Schott. Fibres for use in astronomy are mostly of the multimode type with core diameters between 50 and a few hundred $\mu \mathrm{m}$. The FOCES optical design is built essentially on the assumption that the exit pupil of the microlens serves as the spectrograph entrance slit. This limits the fibre diameter to values $\leq 100 \mu \mathrm{m}$. For a resolution of $R \approx 40000$ a larger diameter would require aperture slicing. For better comparison we have examined fibres with core diameters between 50 and $200 \mu \mathrm{m}$; the data of which are listed in Table 2.

The experimental arrangement is nearly identical to that of Craig et al. (1988), and it therefore differs somewhat from most of the setups found in the literature (e.g. Avila 1988; Ramsey 1988), however, it serves well to at least provide a differential comparison of the fibres. We have taken care that the fibre input is identical to that used in the spectrograph. The fibres are cut, inserted into a holder and polished; the customized fibre ends are then plugged into a precision stage using an adapter. The stage is mounted on a rotating table such that the fibre end is always found on the axis of rotation. A stationary He-Ne laser mounted outside the table illuminates the fibre end with the angle of incidence defined by the angular position of the rotating table. The light cone at the fibre exit is not filled uniformly (except for very small angles of incidence); but its outer edge is well-defined, so the $f$-ratio can
Table 2. Fibres tested with respect to degradation. HCG-MO is from Laser Components, ST and STu are from Mitsubishi, FVP, FHP, and FLP are from Polymicro, and the last three fibres are from Schott. The final column provides the response of the exit light cone to an input of $f / 5.0$

\begin{tabular}{lrrrr}
\hline Type & $\begin{array}{r}\text { Core } \\
(\mu \mathrm{m})\end{array}$ & $\begin{array}{r}\text { Cladding } \\
(\mu \mathrm{m})\end{array}$ & $\begin{array}{r}\text { Buffer } \\
(\mu \mathrm{m})\end{array}$ & $N_{\text {exit }}$ \\
\hline \hline HCG-MO & 100 & 100 & 250 & 4.3 \\
& 200 & 230 & 350 & 4.5 \\
ST & 50 & 300 & 900 & 4.1 \\
& 100 & 400 & 900 & 3.4 \\
STu & 50 & 300 & 900 & 4.0 \\
FVP & 100 & 110 & 125 & 4.8 \\
& 135 & 150 & 165 & 4.5 \\
& 150 & 165 & 195 & 4.6 \\
& 200 & 220 & 240 & 3.7 \\
FHP & 100 & 110 & 125 & 4.7 \\
& 100 & 120 & 140 & 4.9 \\
& 200 & 220 & 240 & 3.5 \\
FLP & 100 & 120 & 140 & 4.6 \\
& 200 & 220 & 240 & 4.0 \\
C5376 & 80 & 200 & 270 & 4.4 \\
C31062 & 100 & 230 & 400 & 4.9 \\
QQ-UV & 200 & 280 & 500 & 4.3 \\
\hline
\end{tabular}

be determined from measurements on an exit screen without ambiguity. From comparison of the results for positive and negative input angles we estimate that our data refer to approximately $80 \%$ of encircled light.

The corresponding output light cones are represented in the last column of Table 2 for an $f / 5$ input from which we conclude that quite generally the $100 \mu \mathrm{m}$ fibres perform much better than the $200 \mu \mathrm{m}$ ones. This does not seem to depend on manufacturer or cladding with the exception that the Mitsubishi $50 \mu \mathrm{m}$ fibres have a degradation even stronger than the $200 \mu \mathrm{m}$ ones. While similar results have been reported by Avila \& D'Odorico (1988) and Guérin \& Felenbok (1988) these data are at variance with the fibre properties published by Ramsey (1988). The outstanding performance of the $100 \mu \mathrm{m}$ fibres is therefore not easy to understand. The Polymicro fibres seem to have the least degradation and were selected for further transmission studies.

Transmission of monochromatic light was measured with a monochromator using the exit slit as spectral resolution element of $\approx 8 \mathrm{~nm}$. Fibres of $25 \mathrm{~cm}$ and $100 \mathrm{~m}$ length, respectively, were homogeneously illuminated using a halogen lamp to measure the relative transmission with a photodiode. Typical results interpolated for $20 \mathrm{~m}$ fibre length are shown in Fig. 4 for the three Polymicro batches. Whereas the well-known $\mathrm{OH}^{-}$absorption features in the near IR are clearly seen in the FVP and FHP fibres the red-sensitive FLP did not show it. This surprising result once more emphasizes the necessity to individually examine the batches. 


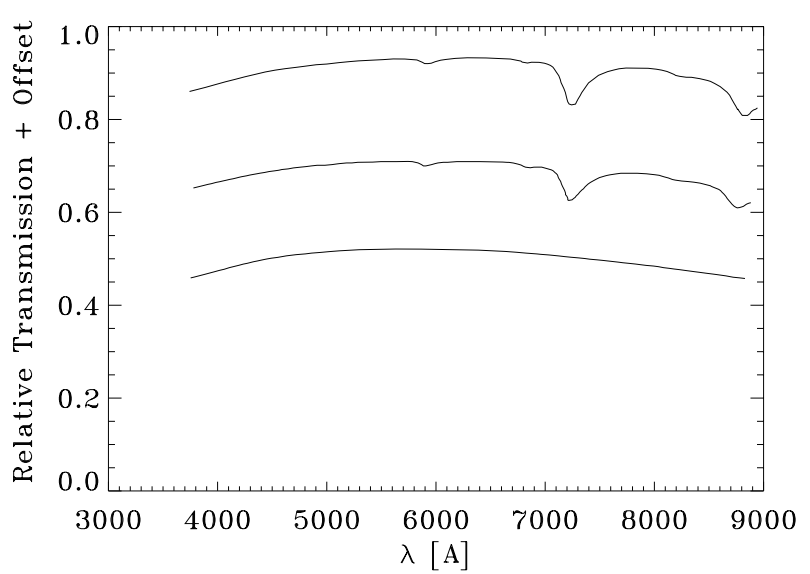

Fig. 4. Spectral transmission of selected $100 \mu \mathrm{m}$ fibres interpolated to a length of $20 \mathrm{~m}$. The fibre types are (from top to bottom): Polymicro FVP, FHP, and FLP. For better comparison they have been offset by 0.2 units

\subsection{Fibre confectioning}

The degradation tests show that typical fibres react sensitively on strain and stresses. Since optical fibres are usually delivered with only a polyamide coat to avoid light losses, long fibre connections suffer from stresses under heavy bending. To avoid extreme degradation it is therefore necessary to put the fibre in a tube that takes most of the strain and stresses from the fibre itself. The FOCES fibres have been confectioned by wrapping the original fibre in a smoothly fitting teflon mantle, and then again wrapping the resulting tube in a steel spiral. As a result the fibres should not suffer very much from focal ratio degradation. However, first observations (Sect. 4) show a residual non-grey noise level of $0.5 \%$ when moving the fibre position with the telescope. Thus at present the spectral properties of the light transfer through the optical fibres must be considered as experimental only.

In spite of the careful confectioning the insertion of the fibre produces differential forces with respect to the tube due to expansion under gravity that can lead to disruption of the fibre. The fibre is therefore carried through a strain compensation box that is attached to the spectrograph immediately in front of the spectrograph slit. In this box the fibre runs in a few uncoated loops which allows for a compensation of the interacting forces. It also makes it possible to thread the fibres into the plugs which otherwise would be a topological problem. Thus the confectioning of the fibres turned out to be one of the most complicated tasks of the project.

\subsection{Telescope module and fibre entry}

The interface between the telescope and the fibre optic input at the focal plane consists of a telescope module
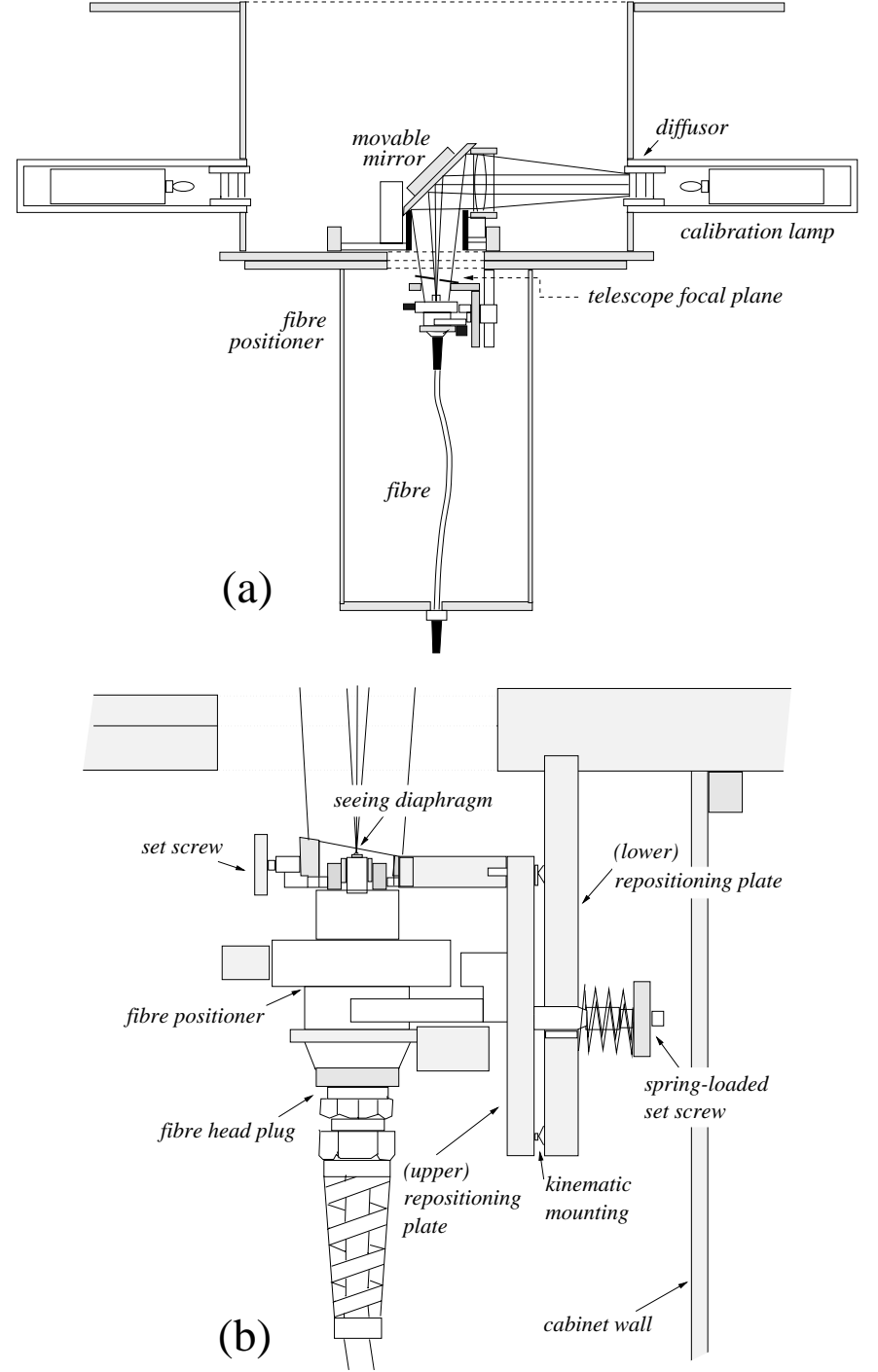

Fig. 5. a) Telescope module with fibre head and calibration lamps. b) Fibre positioning unit

mounted at the Cassegrain flange. It is shown in Fig. 5a. The telescope module carries the the fibre head with a reflecting tilted circular aperture that determines the angular field accepted by the fibre, and simultaneously allows guiding on the entrance aperture using the telescope guiding facilities. It also provides the calibration lamps for flatfield and wavelength comparison exposures.

\subsubsection{Entrance aperture}

Light entering the échelle spectrograph through the fibre passes through a circular diaphragm in the small tilted mirror just atop the fibre head. Three such entrance apertures can be exchanged, with corresponding input light cone (fibre $f$ ratio) and resolution as given in Table 3 . The light reflected from the aperture edges can be observed with the telescope guiding system. 
Table 3. Single-fibre mode entrance apertures and corresponding resolution

\begin{tabular}{rcrrrrr}
\hline Diameter & \multicolumn{2}{c}{ Seeing FWHM } & \multicolumn{2}{c}{ Fibre $f$-ratio } & \multicolumn{2}{c}{ Resolution } \\
$(\mu \mathrm{m})$ & $2.2 \mathrm{~m}$ & $3.5 \mathrm{~m}$ & $2.2 \mathrm{~m}$ & $3.5 \mathrm{~m}$ & $2.2 \mathrm{~m}$ & $3.5 \mathrm{~m}$ \\
\hline 130 & $1.5^{\prime \prime}$ & $0.8^{\prime \prime}$ & 6.2 & 7.7 & 40600 & 50000 \\
200 & $2.3^{\prime \prime}$ & $1.2^{\prime \prime}$ & 4.0 & 5.0 & 26400 & 32500 \\
300 & $3.5^{\prime \prime}$ & $1.8^{\prime \prime}$ & 2.7 & 3.3 & 17600 & 21700 \\
\hline
\end{tabular}

In case of the dual-fibre mode the entrance aperture consists of 2 diaphragms with the same diameters as given above. In the telescope focal plane the holes are separated by $3 \mathrm{~mm}$ corresponding to $35 \operatorname{arcsec}(2.2 \mathrm{~m})$ and 18 arcsec $(3.5 \mathrm{~m})$, respectively. The orientation of the dual-fibre aperture is fixed to E-W direction; it can be changed only by rotating the telescope guide system.

\subsubsection{Calibration lamps}

At present the telescope module provides two calibration light sources that are housed in tubes attached to the module at $90^{\circ}$ distance. Their beams are designed to have roughly the same $f$-ratio as the telescope beam in order to fill the entrance aperture with the same light cone. The actual light source is located at the diffusor the diameter of which also determines the light cone. A field mirror tilted by $45^{\circ}$ with respect to the beam reflects the light onto the diaphragm. It can be moved into and out of the field by a sledge, and it can be rotated by $90^{\circ}$ steps to accept light from either input source. Calibration light sources are a halogen lamp for flatfield exposures and a Th-Ar lamp for wavelength comparison standards. Both lamps and the field mirror are operated remotely from the console.

\subsubsection{Fibre head}

As shown in Fig. 5a the fibre head is attached to the telescope module immediately below the telescope focal plane. Its detailed configuration is shown in Fig. 5b. It consists of a fibre head plug that is plugged into a positioning unit, so that the fibre head plug can be adjusted to its proper position by corresponding slides. The fibre head plug holds the head of the optical fibre and the microlens that is used to re-image the telescope pupil onto the fibre head. The imaging principle is shown in Fig. 6a, from which it is obvious that the diameter of the focal plane aperture determines the $f$-ratio of the light cone entering the fibre. As described in Sect. 4 the available entrance apertures should be chosen with respect to the seeing disk. This particular re-imaging construction of the light path avoids the disadvantages of a direct fibre feed that lead to imperfect scrambling of the object light depending on seeing or, more generally, on the distribution of light in the focal plane (cf. Watson \& Terry 1995).

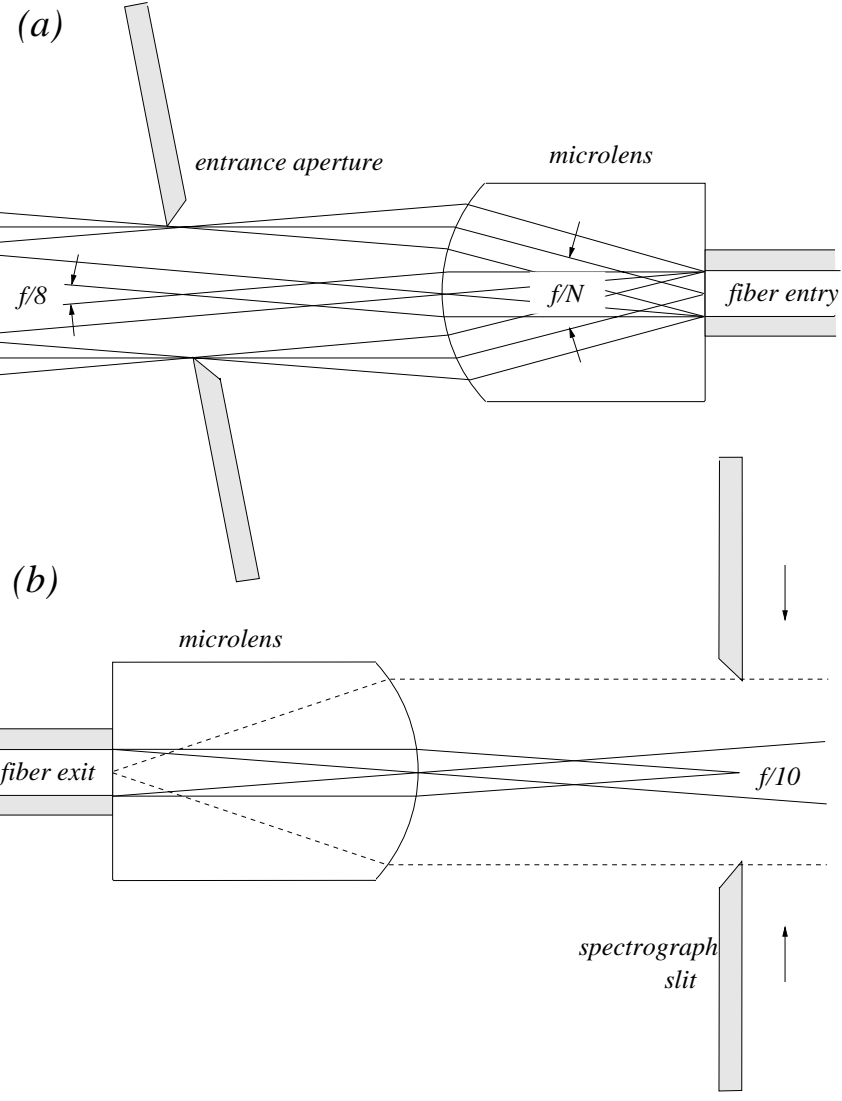

Fig. 6. Fibre feed and exit optics

\subsubsection{Fibre exit and slit assembly}

The fibre exit is optically very similar to the fibre head. It is mounted into the same type of plug and fibre positioner, and it produces a scrambled image of the entrance aperture exactly at the entrance slit of the spectrograph using a microlens glued to the fibre exit surface. The corresponding principle is shown in Fig. 6b. The symmetric optical interfaces at entrance and exit of the fibre also have the advantage that the degradation of the light cone in the fibre does not overly affect the transfer efficiency. Whereas the resolution changes with the slit width, the collimator always produces an image of the full exit pupil on the échelle grating provided that the spectrograph entrance slit is fully open.

This arrangement provides a simple but efficient way to increase the resolution by narrowing the spectrograph entrance slit. The corresponding light losses are substantially smaller than those obtained with a fibre of appropriately reduced diameter or a correspondingly smaller diaphragm at the fibre head. Assuming a Gaussian seeing of full halfwidth $\sigma$, an entrance diaphragm of diameter $\theta$ will pass a fraction of the light of a point source according to

$$
F(\theta) / F(\infty)=1-\exp \left\{-(\ln 2)(\theta / \sigma)^{2}\right\}
$$

with light losses $\Delta$ mag as follows: 


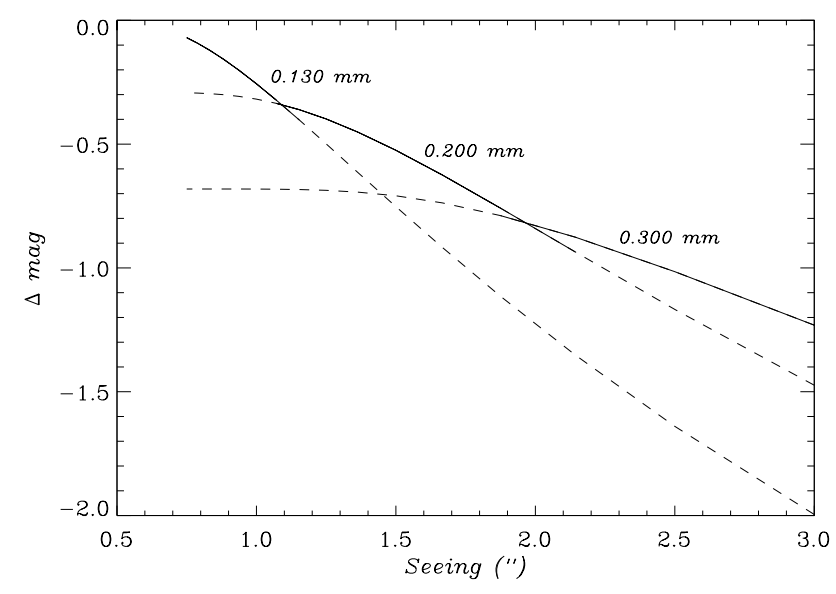

Fig. 7. Light losses at the $2.2 \mathrm{~m}$ telescope at $R=40600$ with different entrance apertures. The choice of the appropriate aperture is indicated by the continuous part of the curves

Table 4. Light losses at the FOCES entrance diaphragm

\begin{tabular}{r||r|r|r|r|r|r|r}
$\theta / \sigma$ & 0.50 & 0.75 & 1.00 & 1.25 & 1.50 & 1.75 & 2.00 \\
\hline$\Delta \mathrm{mag}$ & -2.00 & -1.23 & -0.75 & -0.45 & -0.26 & -0.14 & -0.07
\end{tabular}

The spectrograph slit instead cuts out a rectangular cross-section of width $\phi$ of the entrance aperture diameter $\theta$, where now

$F(\phi) / F(\theta)=1-\frac{2}{\pi}\left\{\arccos x-x \sqrt{1-x^{2}}\right\}$,

and $x=\phi / \theta$. Since $\phi$ determines the spectral resolution the transfer of photons through the fibre system is controlled by the product of the two formulae above. Thus for a given spectral resolution there are three ranges of seeing that require a particular choice of an optimal entrance aperture as shown in Fig. 7 for $R=40600$. Note that the use of the diaphragm with $130 \mu \mathrm{m}$ diameter - although fitting exactly to the resolution - is only suggested for a seeing below 1 arcsec. The larger diaphragms are considerably better for medium or bad seeing. Of course, no light is lost even with bad seeing if the resolution is adjusted accordingly by opening the spectrograph slit.

\section{First observations}

FOCES has been through a number of test observing runs in the laboratory and on three telescopes, the $80 \mathrm{~cm}$ Wendelstein telescope of the Munich University Observatory, and the DSAZ $2.2 \mathrm{~m}$ and $3.5 \mathrm{~m}$ telescopes on Calar Alto. In addition to tests of proper installation and fine adjustment of the optical components a number of stellar spectra were taken ranging from $V \approx 0$ (Procyon) to $11 \mathrm{mag}$. Weather conditions at the telescopes were never near to optimal with both seeing and atmospheric transparency degrading the results; therefore all estimates of the spectrograph performance are somewhat preliminary.
The instrument parameters are under computer control except for the spectrograph slit which is adjusted manually. They are operated from a VT100-type terminal emulation, and they provide spectrograph settings, object identification, and exposure with a number of submenus.

\subsection{FOCES data reduction software}

The FOCES échelle data reduction software (EDRS) consists of a number of modules written in IDL (Version 4$)^{1}$. Such modules comprise the basic input/output and datahandling routines, display functions that allow inspection of the original and reduced data, auxiliary routines which support detection of features and image processing, and échelle spectrum extraction. In addition to the basic modules there exist application command files to extract wellcalibrated one-dimensional spectra in a fully automatic mode. The single routines contain entry and exit variables (arguments), and optional keyword parameters. Since the language is interpreted, the FOCES software package consists of source code. All procedures or functions include a documentation header that can be read online with an INFO command. All efforts have been undertaken to make the EDRS software package as independent of operating system features as is possible under IDL, however, the principal use is currently under a DIGITAL UNIX system. No attempts have been made to generalize the EDRS software for use with data obtained from other spectrographs.

FOCES spectrum extraction is subdivided into a number of consecutive steps for which a standard set of exposures must be available if the automatic extraction mode is to be used successfully. These exposures must all refer to the same set of parameters such as CCD camera and center wavelength setting etc. If only one of the above exposures is missing the automatic extraction will not give the desired result. The EDRS automatic data reduction is able to co-add or average object, bias, dark, flatfield or calibration exposures. Bias and dark subtraction are done as usual leaving only the true spectrographic images, corrected for much of the instrumental response. Additional parameters determining details of the spectrum extraction process are entered by editing a FITS-like parameter file, USER.PAR. The following subsections describe the reduction steps.

\subsubsection{Order detection}

The primary detection routine, ORD_PATT, finds the approximate positions of the échelle orders by using crossorder scans starting at the center of order dispersion where the signal is highest. From there it follows the order ridges

${ }^{1}$ IDL is a high-level interactive language distributed by Research Systems Inc. 


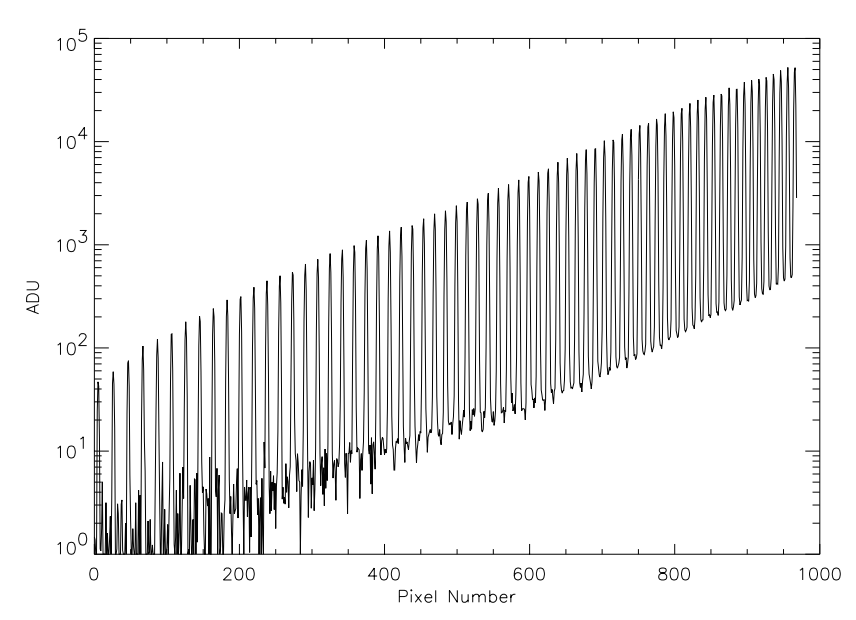

Fig. 8. Cross-order intensity tracing over the whole wavelength range. Note the logarithmic scale. Order \# 145 (left) corresponds to $3950 \AA$, order \# 78 (right) to $7340 \AA$. Order separation is between 20 pixels in the blue and 10 pixels in the red. The straylight background is everywhere less than $1 \%$ of the adjacent order signal

to both sides, and it approximates the order ridge positions by polynomials of low degree. This procedure is reasonably robust against hot pixels and bad columns since it first applies a median filter to the image which does not affect the search for the true position of the order ridges. Since the object exposures are normally faint, the order positions should be obtained from the corresponding order flatfield exposure instead. The above algorithm works on continuous orders; it fails to detect the order positions of wavelength calibration exposures and of pure emission line objects.

\subsubsection{Background correction}

Even the best spectrograph cannot completely avoid scattered light. This is found between the spectral orders at an intensity level sometimes significantly above zero. However, for FOCES this light level is very low as is demonstrated in Fig. 8 which also shows that the interorder minimum signal is uniformly proportional to the order maximum signal if the intermediate slit is in place. Due to the narrow cross-order intensity profile $(\approx 3$ pixels) the crowding of the orders in the red does not lead to a strong increase of the interorder minimum signal. Using the model of Gehren \& Ponz (1986) assuming a local straylight origin, the contribution to the extracted order spectrum is between $0.9 \%$ in the blue and $1.8 \%$ in the red. This is roughly proportional to the inverse order separation. Simple subtraction of the interorder minimum intensity represents a global straylight approximation, and it leads to a contribution that increases with order separation; this would suggest much higher straylight contamination of between $5.2 \%$ in the blue and $2.6 \%$ in the red.

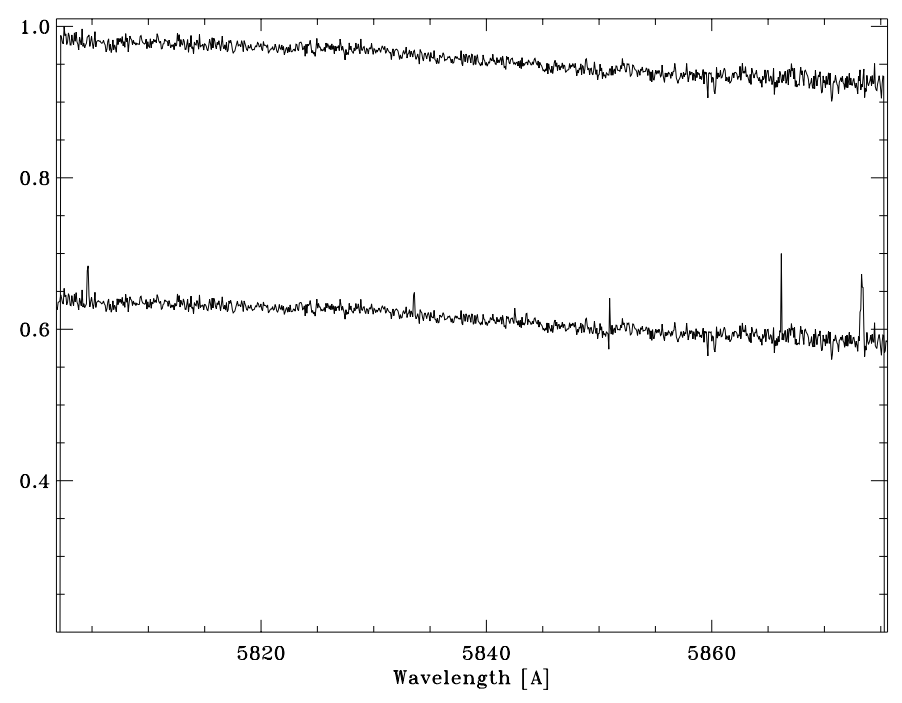

Fig. 9. Difference between the results of RAW_ORDER and OPT_EXTR demonstrating essentially the removal of cosmic events. The two spectra are offset by a constant amount

Since in the red (where the signal is high) both methods differ by less than $1 \%$ it is not evident from tests which of the two methods yields the more consistent results. At present the interorder minimum subtraction is therefore preferred. We note that removing the intermediate slit increases the background straylight by both a global component and a local component that is increased by a factor $>2$.

\subsubsection{Order extraction}

Once the order positions are known the appropriate order extraction is applied to all remaining échelle images (object, order flatfield, and wavelength comparison). There are basically two ways to sample the échelle orders, one using the order ridge positions $y^{(m)}(x)$ and a specified sampling width $\Delta y, y$ being the cross-order coordinate. With these data the pixel intensities found at constant $x$ within $y^{(m)}(x) \pm \Delta y / 2$ are added to define the spectrum order flux. This type of extraction is obtained with procedure RAW_ORDER. The second procedure uses an elaborate method to estimate the intensity values found across the order. This allows to detect non-linear pixel intensities by comparison with a mean cross-order profile, and it is done using an optimal extraction algorithm in procedure OPT_EXTR similar to the one proposed by Horne (1986).

After order extraction has been performed, the order spectra still contain the échelle blaze which is essentially a $(\sin x)^{2} / x^{2}$ function. The blaze is mostly removed by dividing the object exposure through the extracted (onedimensional) order flatfield. Since the order flatfield spectrum is exposed through the same fibre as the object, it should leave the resulting object continuum modulated with the ratio of the continuous spectra of object and 


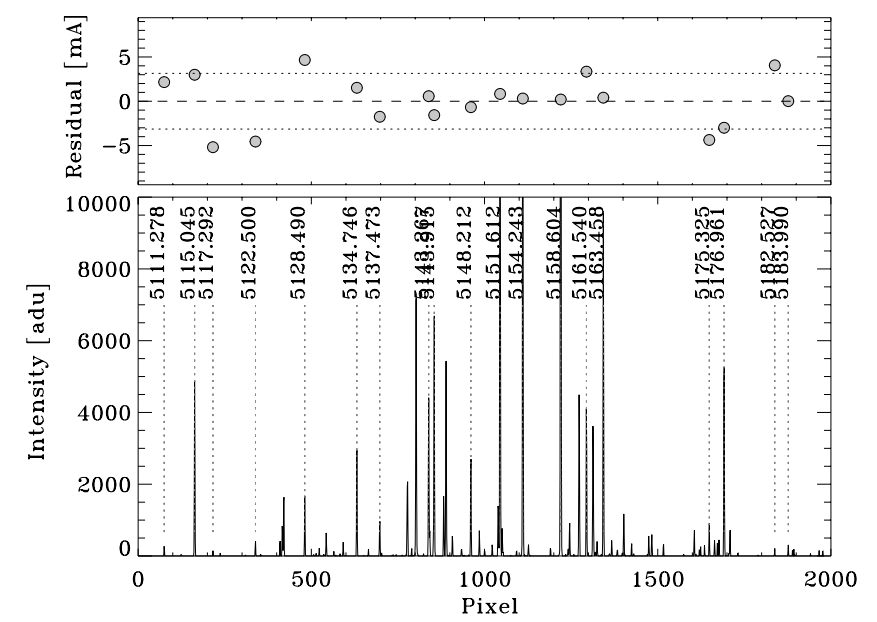

Fig. 10. Accuracy of wavelength calibration in a single order obtained with the Tektronix $2048^{2}$ chip with $24 \mu \mathrm{m}$ pixel distance

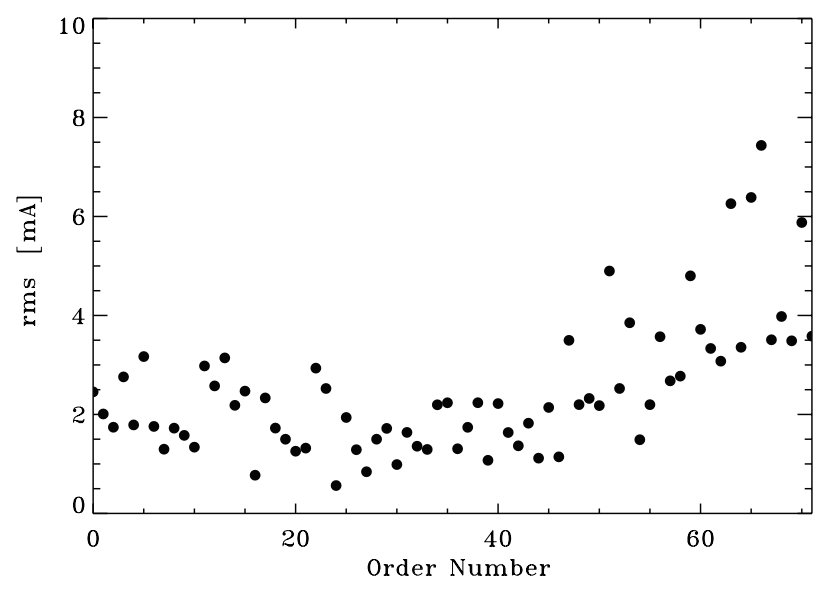

Fig. 11. Rms error in $\mathrm{m} \AA$ of wavelength calibration obtained with the Tektronix $1024^{2}$ chip with $24 \mu \mathrm{m}$ pixel distance varying with order number. Here, order \#0 is in the blue (4170 $\AA$ ), order \#71 in the red (8850 ̊)

flatfield lamp. Figure 9 shows that for sufficiently high signal the difference between the two extraction modes mentioned above is mostly due to the different treatment of cosmic ray events.

\subsubsection{Wavelength calibration}

WAVE_CAL provides the wavelength calibration of the extracted order spectra; first it compares the extracted multi-order comparison spectrum with a template spectrum that consists of a multi-order intensity tracing combined with an appropriate wavelength calibration. Centering of the order spectra with respect to the $x$ coordinate as well as the correct identification of the order pattern (with the center wavelength) is achieved using a very simple version of a spatial correlation function. A nearly perfect calibration of the new spectrum is thus already obtained as a first approximation. A calibration spectrum line list containing a sufficient number of emission lines is then compared with lines detected on the calibration exposure. The emission line maximum positions are found with a maximum parabola fit procedure. The solution is iteratively improved excluding all deviations of single line positions $>n \sigma$ (the default of $n$ is 3 ), as long as there are still enough lines left. The minimum number of lines left is restricted by the degree of the polynomial approximation. At the end of the calibration procedure the extracted wavelength calibration lamp fluxes are replaced by the wavelengths at the corresponding pixel positions. The results of this process are displayed in Fig. 10 for a single order. The high accuracy of $0.15 \mathrm{~km} \mathrm{~s}^{-1}$ obtained in a single order confirms the expected performance of the spectrograph with the $24 \mu \mathrm{m}$ Tektronix chip. Figure 11 demonstrates that the rms mean calibration error of all orders is just as low although there exists a small degradation towards the red. Experience shows that the Loral $2048^{2}$ chip with $15 \mu \mathrm{m}$ pixel distance provides a similar accuracy. A single order rms error of $2.5 \mathrm{m \AA}$ in the visible spectrum corresponds to an accuracy of $150 \mathrm{~m} \mathrm{~s}^{-1}$; the formal error of the 70 order mean is only $18 \mathrm{~m} \mathrm{~s}^{-1}$. The latter value is reproduced for a number of exposures, and it verifies the expectactions of instrumental accuracy. This compares favourably with the results found for the ELODIE spectrograph of Baranne et al. (1996).

\subsection{Comparison of predicted and actual performance}

The performance of the spectrograph was originally estimated to be around $13 \%$, using as much as possible the specifications given by the corresponding instrument data sheets including mirrors, fibres, prisms, lenses, and the CCD. This is degraded by two telescope mirror reflections to roughly $10 \%$. Thus assuming ideal observing conditions with no atmospheric extinction and a seeing below 1 arcsec, the limiting performance in the $V$ band should yield 40 photons per second and 2-pixel resolution element for a 10th magnitude star. It was mentioned above (see Fig. 3) that the seeing was mostly poor on Calar Alto. This implies that under normal seeing conditions a considerable fraction of the photon flux is lost as is shown in Fig. 7. Whenever full resolution is required the possible faintest magnitude thus will be affected by $\approx 0.3-1 \mathrm{mag}$. Own experience during the test runs confirms this estimate: for an average seeing of $2-3$ arcsec the limiting magnitude - obtained for a 1 hour stellar exposure that produces a $S / N$ of 100 with a resolution of 40000 at the DSAZ $2.2 \mathrm{~m}$ telescope - was around $V=11.2$, corresponding to only $42 \%$ of the theoretical performance. With improved seeing of only 1 arcsec the limiting magnitude would increase to $V=11.8$. The atmospheric transparency has not yet been considered; thus an optimal value for the limiting magnitude might be as high as $V=12.1$. This is only a few percent below our initial theoretical estimate, and it would 


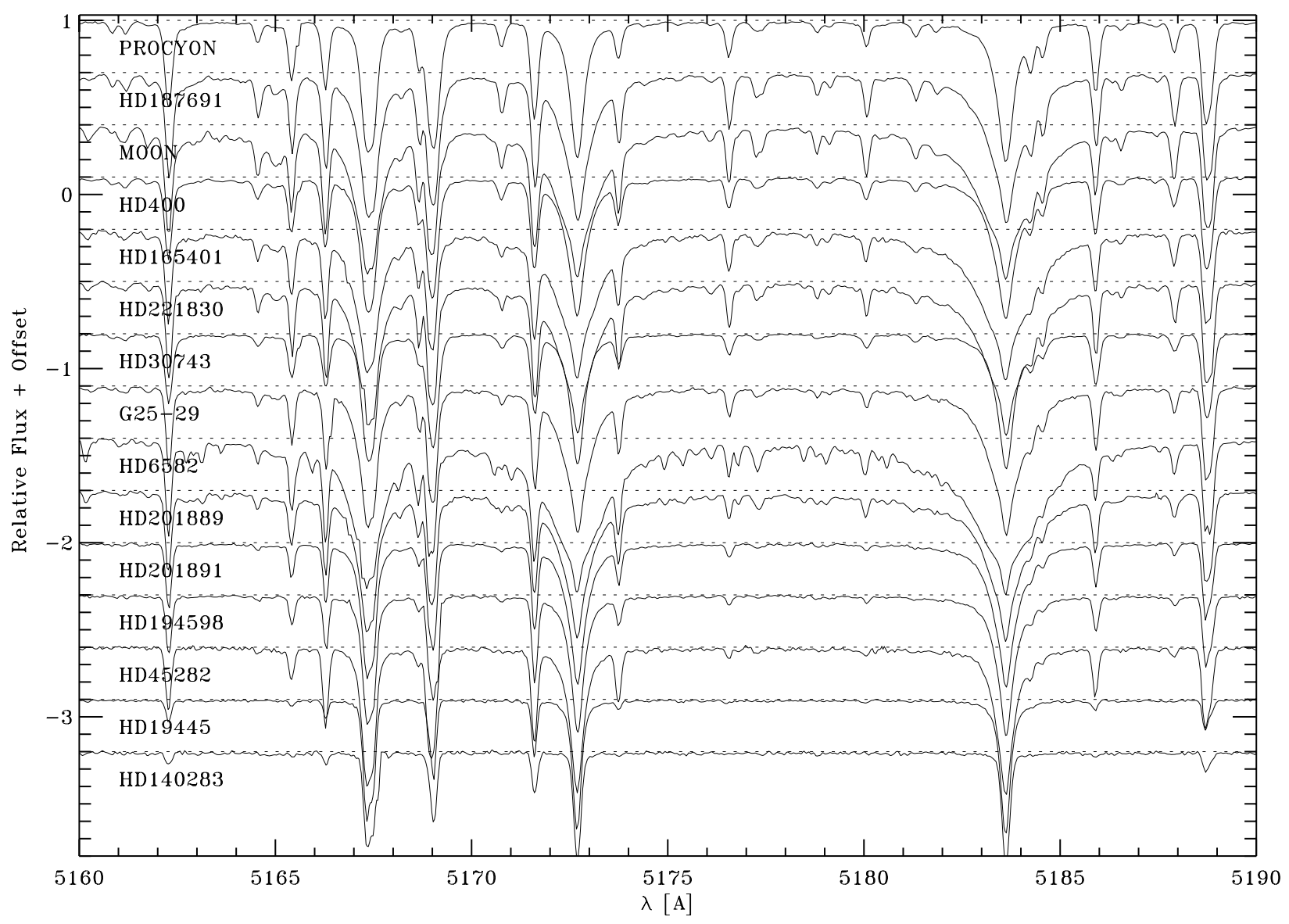

Fig. 12. Selected spectra of cool stars observed during test runs. Stars are ordered roughly according to decreasing metal abundance. The spectral region includes the $\mathrm{Mg}$ Ib lines with some strong contributions of $\mathrm{C}_{2}$ and $\mathrm{MgH}$, particularly strong in cool stars such as HD 6582

confirm our theoretical model parameters. With the seeing improved according to Table 3 the limiting magnitude for the $3.5 \mathrm{~m}$ telescope would be $1.0 \mathrm{mag}$ higher, however, as shown in Table 4 light losses due to inadequate seeing may easily compensate the increased collecting area of the larger primary mirror.

On the $1024^{2}$ Tektronix CCD the image of the échelle spectrum covers approximately 70 orders in the visual with full overlap to the first minimum of the blaze function up to $\approx 5200 \AA$. A typical exposure is displayed in Fig. 3. During the first test observing runs a total of more than 200 exposures for 73 stars have been obtained. A small part of some of the typical spectra around the $\mathrm{Mg} \mathrm{Ib}$ lines is shown in Fig. 12. As is evident from Fig. 9 the spectral continuum is well defined. Thus it is possible to measure line profiles such as the $\mathrm{Mg}$ Ib lines to an accuracy better than $1 \%$ (under most circumstances even better than $0.5 \%$ ).

One of the main drivers of fibre-fed spectrographs installed outside the telescope dome is the absence of any kind of mechanical or thermal irregularities, provided that the spectrograph laboratory environment is under control.
This should guarantee very small residuals in wavelength calibration as well as a high internal accuracy of the dispersion scale. This was in fact observed during the test runs. The results are reproduced in Fig. 10. The accuracy of the dispersion calibration (obtained in the fully automatic mode) can be estimated in particular from Fig. 11 which produces a mean error of the complete échelle spectrum of less than $20 \mathrm{~m} \mathrm{~s}^{-1}$. Comparison of multiple exposures confirms that not only internal velocity differences are rather small but also the absolute values are highly accurate.

During the first test observations the resolution was still limited by the CCD pixel size (i.e. $24 \mu \mathrm{m}$ for the Tektronix chip). The corresponding resolution limit is nicely demonstrated by comparing spectra of a single star and a binary in Fig. 13. Here, the faintest lines are just being resolved, and the binary nature of G28-43 becomes evident. Since the actual resolution product of FOCES is near $R \phi=60000$, the resolution can be improved at the expense of throughput using a $2 \mathrm{k}$ chip with $15 \mu \mathrm{m}$ pixels and a correspondingly narrower slit without installing a longer camera. A few more recent observations have been 


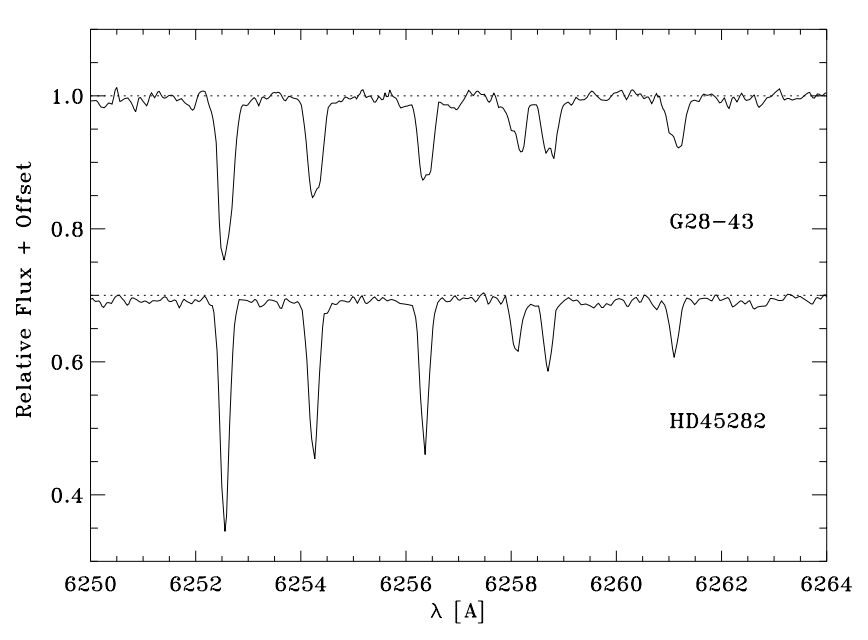

Fig. 13. Spectral window of 2 metal-poor stars of similar stellar parameters. HD 45282 (bottom) displays maximum resolution ( $R=40600$ ) with a number of medium to faint Fe I and Ti I lines. G28-43 (top) emerges to be an SB2-type binary, just at the resolution limit

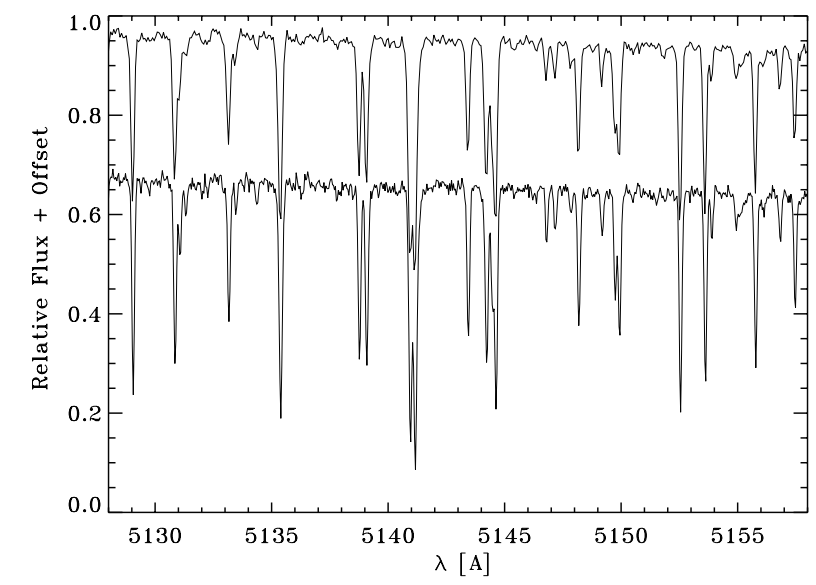

Fig. 14. Spectra of HD 22879 taken in two exposures with the $24 \mu \mathrm{m}$ Tektronix CCD (top), and with the $15 \mu \mathrm{m}$ Loral CCD (bottom), respectively; the entrance slit was adjusted to fit exactly 2 pixel resolution elements such that $R=40600$ (top) and $R=65000$ (bottom). The bottom spectrum is shifted by a constant amount

taken at the $2.2 \mathrm{~m}$ telescope with $R=65000$ using the Loral $2048^{2}$ CCD. Comparing the different exposures in Fig. 14 the increase in resolution is evident from both line halfwidth and blend separation. We note that with the increased area of the Loral CCD the spectral coverage is extended to 3800 - $9200 \AA$.

A final experiment was the installation of the dualfibre mode intended to be used for background subtraction from faint object signals such as obtained at the $3.5 \mathrm{~m}$ telescope. The fibre head consists of two identical diaphragms with $3 \mathrm{~mm}$ distance corresponding to $18^{\prime \prime}$. The position angle can be adjusted rotating the telescope guide unit if necessary. Both diaphragms feed identical $100 \mu \mathrm{m}$ fibres through microlenses, and both fibres are aligned on the spectrograph slit. Thus the échelle pattern is double; be- tween each pair of object spectral orders the corresponding sky background order is placed.

The cross dispersion required for the dual-fibre mode is approximately twice as high as in the single fibre mode since camera and detector are the same as in the standard single-fibre mode. It is provided by an additional blue or red grism placed in the beam immediately in front of the double prism. Present observational experience has not yet produced enough evidence to precisely describe the performance of the dual-fibre system.

FOCES has been in regular use now at the $2.2 \mathrm{~m}$ telescope for a number of observing runs dealing mostly with high-resolution stellar spectroscopy. However, the crossorder flatfield and the optimal fibre configuration will have to be improved.

Acknowledgements. The FOCES project was generously supported by grants 05 SMU414(8) and 05 2MU114(7) obtained from the German ministry of science and technology (BMFT). We are particularly grateful to G. Avila, H. Dekker, B. Delabre, and S. D'Odorico of the European Southern Observatory, Garching, who supported our spectrograph project from the beginning, and who worked out much of the final optical design; to K. Birkle, H. Elsässer, K.H. Marien, W. Rauh, J. Solf, U. Thiele and K. Wagner of the Max-Planck-Institut für Astronomie in Heidelberg, who helped us find a working compromise between a fully autonomous instrument and one that can be scheduled for the typical astronomer.

\section{References}

Avila G., 1988, in Optical Fibres in Astronomy, ASP Conf. Ser. 3, Barden S.C. (ed.). San Francisco, p. 63

Avila G., D'Odorico S., 1988, in Very Large Telescopes and their Instrumentation, Ulrich M.-H. (ed.). ESO, Garching, p. 1121

Barden S.C., 1995, in Fiber Optics in Astronomical Applications, SPIE Proc. 2476, p. 2

Baranne A., 1988, in ESO Conference on Very Large Telescopes and their Instrumentation, Ulrich M.-H. (ed.). Garching, p. 1195

Baranne A., Queloz D., et al., 1996, A\&AS 119, 373

Craig W.W., Hailey C.J., Brodie J.P., 1988, in Optical Fibres in Astronomy, loc.cit., p. 41

Dekker H., Delabre B., et al., 1992, in Progress in Telescope and Instrumentation Technologies, Ulrich M.-H. (ed.). ESO, Garching, p. 581

Gehren T., Ponz J., 1986, A\&A 168, 386

Guerin J., Felenbok P., 1988, in Optical Fibres in Astronomy, loc.cit., p. 52

Heacox W.D., 1986, AJ 92, 219

Hall J.C., Fulton E.E., et al., 1994, PASP 106, 315

Horne K., 1986, PASP 98, 609

Ramsey L.W., 1988, in Optical Fibres in Astronomy, loc.cit., p. 26

Ramsey L.W., Huenemoerder D.P., 1987, SPIE 621, 282

Schroeder D.J., 1987, Astronomical Optics. Academic Press, San Diego

Watson F.G., Terry P., 1995, in Fiber Optics in Astronomical Applications, loc.cit., p. 10 